\title{
A RIESZ REPRESENTATION THEOREM
}

\section{ROBERT KENT GOODRICH}

Introduction. This paper deals with a Riesz representation theorem in the following setting: Let $H$ be a compact Hausdorff space, let $E$ and $F$ be locally convex Hausdorff topological vector spaces over the real or complex field. Let $C(H, E)$ denote the continuous functions from $H$ into $E$ with the topology of uniform convergence. The purpose of this paper is to improve certain integral representation theorems for continuous linear transformations $T$ from $C(H, E)$ into $F$.

The well-known Riesz representation theorem [10] gives a Stieltjes integral representation for $T$ when $H$ is a closed interval and when $E$ and $F$ are the real numbers. There have been many generalizations of this theorem in the literature, and there have been two essentially different approaches giving rise to two different kinds of representation theorems. In one approach (see [1], [2], [5], [8], [9], [11]) $y^{\prime} T$ is written as an integral where $y^{\prime}$ is in the topological dual of $F$. The integral converges in the weak topology with a measure defined on the Borel sets with values in $L\left(E, F^{\prime \prime}\right)$, the space on continuous linear transformations of $E$ into $F^{\prime \prime}$. Under various additional assumptions on $E, F$, or $T, T$ is written as an integral. (For example, this can be done if $T$ is assumed to be compact or $F$ is assumed to be reflexive.) The most general of these theorems is due to Swong [11, Theorem 6.1, p. 283]. Another approach in the literature can be found in the papers [6], [12], [13], [14]. In these papers the method is of a constructive nature and $T$ is written as an integral, and the convergence of the in tegral is in the $\epsilon^{\circ 0}$ topology of $F^{\prime \prime}$ (norm topology in the case $F$ is a normed space), and $T$ is thought of as a mapping of $E$ into $F^{\prime \prime}$ by the canonical imbedding of $F$ into $F^{\prime \prime}$. This is done by restricting the class of sets the measure is defined on. In [12] the class of sets is the intervals where $H=[0,1]$, and $E$ and $F$ are assumed to be normed spaces. In [6] and [13] the class of sets is the ring generated by compact $G_{\delta}$ 's. Recently in [14] Tucker and Wayment have been able to extend the measure to a larger proper subring of the Baire sets.

In this paper it is shown that the restriction of the measure in [6], [12], [13], [14] was not necessary, and in fact $T$ can be represented as an integral with convergence in the $\epsilon^{\circ \circ}$ topology with the measure defined on the Borel sets. This result thus implies and strengthens

Received by the editors June 20,1968 and, in revised form, June 12, 1969. 
each of these previous results. Further, the proof is new and somewhat simpler.

The second problem considered is the special case when $E$ is the scalars and $F=L(X, Y)$ and $X$ and $Y$ are linear normed spaces. It is shown that the measure can be taken with values in $L\left(X, Y^{\prime \prime}\right)$ instead of $L^{\prime \prime}(X, Y)$ as assured by our first result.

The third problem considered is the case when $H$ is also a topological group and we characterize the left translation invariant $T$.

1. Notation. The topology of $C(H, E)$ is generated by the seminorms $p(f)=\sup _{t \in H} p(f(t))$ where $\{p\}$ ranges over the continuous seminorms of $E$. Let $C(H)$ denote the continuous functions from $H$ into the scalar field with the usual supremum norm. Let $L(E, F)$ denote the continuous linear transformations from $E$ into $F$.

If $G$ is any locally convex Hausdorff topological vector space then let $G^{\prime}$ denote the topological dual of $G$ under the topology of uniform convergence on weakly bounded subsets of $G$. Let $G^{\prime \prime}$ denote the dual of $G^{\prime}$. The topology for $G^{\prime \prime}$ will be the $\epsilon^{\circ 0}$ topology which is generated by the seminorms $q^{\prime \prime}\left(g^{\prime \prime}\right)=\sup _{g^{\prime} \in B_{a}^{0}}\left|\left\langle g^{\prime \prime}, g^{\prime}\right\rangle\right|$, where $B_{q}$ is the $q$-unit ball for a continuous seminorm $q$ on $G$, and $B_{q}^{0}$ is the polar of $B_{q}$ in $G^{\prime}$, i.e., $B_{q}^{0}=\left\{g^{\prime} \in G^{\prime \prime}:\left|\left\langle g, g^{\prime}\right\rangle\right| \leqq 1, \forall g \in B_{q}\right\}$.

We shall make use of some notation used in Swong's paper [11]. When we say "there exists a pairing $(p, q)$ such that ..." we shall mean "for each continuous seminorm $q$ on $F$ there exists a continuous seminorm $p$ on $E$ such that ..." For example, $T$ is continuous if and only if there exists a pairing $(p, q)$ such that $|T|_{p-q}$ $=\sup \left\{q(T(f)): \sup _{t \in H} p(f(t)) \leqq 1\right\}$ is finite, and whenever $|T|_{p-q}$ exists we shall say $T$ is $p-q$ related. We further notice that $T^{\prime \prime}$ (the bitranspose of $T)$ maps $C^{\prime \prime}(H, E)$ into $F^{\prime \prime}$, and whenever $T$ is $p-q$ related then $T^{\prime \prime}$ is $p^{\prime \prime}-q^{\prime \prime}$ related and $|T|_{p-q}=|T|_{p^{\prime \prime}-q^{\prime \prime}}$.

Let $S$ denote the Borel sets, i.e., the $\sigma$-ring generated by closed subsets of $H$. If $e \in S$ we denote the characteristic function of $e$ by $\chi_{e}$. If $e \in S$ and $x \in E$ we think of $\chi_{e} \cdot x$ as an element of $C^{\prime \prime}(H, E)$ by the following identification: if $f^{\prime} \in C(H, E)$ then $\phi$ defined by $\langle f, \phi\rangle$ $=\left\langle f \cdot x, f^{\prime}\right\rangle$ for $f \in C(H)$ is in $C^{\prime}(H)$. Thus there exists a unique regular Borel measure $\mu_{\left(x, f^{\prime}\right)}$ such that $\phi(f)=\int f d \mu_{\left(x, f^{\prime}\right)}$. Now define $\left\langle\chi_{e} \cdot x, f^{\prime}\right\rangle$ $=\mu_{\left(x, f^{\prime}\right)}(e) \cdot \chi_{e} \cdot x \in C^{\prime \prime}(H, E)$ since it is in the bipolar of the bounded set $A=\{f \cdot x ;\|f\| \leqq 1\}$. For if $f^{\prime} \in A^{0}$ then $\left|\left\langle\chi_{e} x, f^{\prime}\right\rangle\right|=\left|\mu_{\left(x, f^{\prime}\right)}(e)\right|$ $\leqq\left\|\mu_{\left(x, f^{\prime}\right)}\right\|$ (variation of $\left.\mu_{\left(x, f^{\prime}\right)}\right)$. But

$$
\left\|\mu_{\left(x, f^{\prime}\right)}\right\|=\|\phi\|=\sup _{\|f\| \leqq 1}|\langle f, \phi\rangle|=\sup _{\|f\| \leqq 1}\left|\left\langle f \cdot x, f^{\prime}\right\rangle\right| \leqq 1
$$

since $f^{\prime} \in A^{0}$. Thus $\chi_{e} \cdot x \in C^{\prime \prime}(H, E)$. 
2. The representation theorem. We first prove the following lemma:

Lemma 1. If $\left\{e_{1}, \cdots, e_{n}\right\}$ is a partition of $H$ into Borel sets and $x_{1}, \cdots, x_{n}$ are points in $E$, then $p^{\prime \prime}\left(\sum_{j=1}^{n} \chi_{e_{i}} x_{i}\right) \leqq \max p\left(x_{i}\right)$.

Proof. $p^{\prime \prime}\left(\sum_{i=1}^{n} \chi_{e_{i}} \cdot x_{i}\right)=\sup _{f^{\prime} \in B_{p}^{0}}\left|\sum_{i=1}^{n}\left\langle\chi_{e_{i}} \cdot x_{i}, f^{\prime}\right\rangle\right|$. Fix $f^{\prime} \in B_{p}^{0}$, then $\left|\sum_{i=1}^{n}\left\langle\chi_{e_{i}} \cdot x_{i}, f^{\prime}\right\rangle\right|=\left|\sum_{i=1}^{n} \mu_{i}\left(e_{i}\right)\right|$ where $\mu_{i}=\mu_{\left(x_{i}, f^{\prime}\right)}$. By construction each $\mu_{i}$ is a regular Borel measure of finite variation, so for each $i$ pick a compact set $c_{i}$ such that $c_{i} \subset e_{i}$, and $\left\|\mu_{i}\right\|\left(e_{i}-c_{i}\right)<\epsilon / 3 n$. Since $H$ is a compact Hausdorff space, and the $\left\{c_{i}\right\}$ are disjoint, there exist disjoint open sets $\left\{u_{i}\right\}$ such that $c_{i} \subset u_{i}$ and $\left\|\mu_{i}\right\|\left(u_{i}-c_{i}\right)<\epsilon / 3 n$, for all $i$. Now pick compact $G_{\delta}^{\prime}$ s $\left\{c_{i}^{\prime}\right\}$ such that $c_{i} \subset c_{i}^{\prime} \subset u_{i}$. Then

$$
\begin{aligned}
&\left|\sum_{i=1}^{n} \mu_{i}\left(e_{i}\right)-\sum_{i=1}^{n} \mu_{i}\left(c_{i}^{\prime}\right)\right| \\
& \leqq\left|\sum_{i=1}^{n} \mu_{i}\left(e_{i}\right)-\sum_{i=1}^{n} \mu_{i}\left(c_{i}\right)\right|+\left|\sum_{i=1}^{n} \mu_{i}\left(c_{i}\right)-\sum_{i=1}^{n} \mu_{i}\left(u_{i}\right)\right| \\
&+\left|\sum_{i=1}^{n} \mu_{i}\left(u_{i}\right)-\sum_{i=1}^{n} \mu_{i}\left(c_{i}^{\prime}\right)\right| \leqq \sum_{i=1}^{n}\left\|\mu_{i}\right\|\left(e_{i}-c_{i}\right) \\
&+\sum_{i=1}^{n}\left\|\mu_{i}\right\|\left(u_{i}-c_{i}\right)+\sum_{i=1}^{n}\left\|\mu_{i}\right\|\left(u_{i}-c_{i}^{\prime}\right)<\epsilon .
\end{aligned}
$$

Thus $\left|\sum_{i=1}^{n} \mu_{i}\left(e_{i}\right)\right| \leqq \epsilon+\left|\sum_{i=1}^{n} \mu_{i}\left(c_{i}^{\prime}\right)\right|$.

Now for each $i$ pick a sequence $\left\{f_{k i}\right\}$ of continuous real valued functions such that $0 \leqq f_{k i} \leqq 1, \forall k, i$; the support of $f_{k i}$ is in $u_{i}$ for all $i$ and $k ;\left\{f_{k i}\right\}$ converges monotonically downward to $\chi_{c_{i}^{\prime}}$ for each $i$. Then $\left|\sum_{i=1}^{n} \mu_{i}\left(c_{i}^{\prime}\right)\right|=\left|\lim _{k} \sum_{i=1}^{n} \int f_{k i} d \mu_{i}\right|$ by the Lebesgue dominated convergence theorem. But since $f^{\prime} \in B_{p}^{0}$,

$$
\begin{aligned}
\left|\lim _{k} \sum_{i=1}^{n} \int f_{k i} d \mu_{i}\right| & =\lim _{k}\left|\left\langle\sum_{i=1}^{n} f_{k i} \cdot x_{i}, f^{\prime}\right\rangle\right| \leqq \varlimsup_{k} p\left(\sum_{i=1}^{n} f_{k i} x_{i}\right) \\
& \leqq \varlimsup_{k} \sup _{t \in H} p\left(\sum_{j=1}^{n} f_{k i}(t) \cdot x_{i}\right) \\
& \leqq \varlimsup_{k} \sup _{t \in H}\left(\sum_{i=1}^{n} f_{k i}(t)\right) \max p\left(x_{i}\right) \leqq \max p\left(x_{i}\right),
\end{aligned}
$$

since $\sum_{i=1}^{n} f_{k i}(t) \leqq 1$ for all $t \in H$ and all $k .\left|\left\langle\sum_{i=1}^{n} \chi_{e_{i}} \cdot x_{i}, f^{\prime}\right\rangle\right| \leqq \epsilon$ $+\max p\left(x_{i}\right) \forall \epsilon$, and $\forall f^{\prime} \in B_{p}^{0}$. Thus $p^{\prime \prime}\left(\sum_{i=1}^{n} \chi_{e_{i}} \cdot x_{i}\right) \leqq \max p\left(x_{i}\right)$.

Now define $K(e) \cdot x=T^{\prime \prime}\left(\chi_{e} \cdot x\right) . K$ is of bounded $p-q^{\prime \prime}$ variation which means there exists a pairing $\left(p, q^{\prime \prime}\right)$ and constants $W_{p-q^{\prime \prime}}$ such 
that $q^{\prime \prime}\left(\sum_{i=1}^{n} K\left(e_{i}\right) \cdot x_{i}\right) \leqq W_{p-q^{\prime \prime}} \sup \left(x_{i}\right)$. The $p-q^{\prime \prime}$ variation $V_{p-q^{\prime \prime}}$ is defined to be the inf of the constants $W_{p-q^{\prime \prime}}$. To check this take $q^{\prime \prime}$ on $F^{\prime \prime}$ then there exists a $p$ such that $T$ is $p-q$ related and so $T^{\prime \prime}$ is $p^{\prime \prime}-q^{\prime \prime}$ related so

$$
\begin{aligned}
q^{\prime \prime}\left(\sum_{i=1}^{n} K\left(e_{i}\right) \cdot x_{i}\right) & =q^{\prime \prime}\left(T^{\prime \prime}\left(\sum_{i=1}^{n} \chi_{e_{i}} \cdot x_{i}\right)\right) \\
& \leqq|T|_{p^{\prime \prime}-q^{\prime \prime}} p^{\prime \prime}\left(\sum_{k=1}^{n} \chi_{e_{i}} \cdot x_{i}\right) \leqq|T|_{p-q} \max p\left(x_{i}\right) .
\end{aligned}
$$

Thus $K$ is of bounded $p-q^{\prime \prime}$ variation, and in fact $V_{p-q^{\prime \prime}} \leqq|T|_{p-q}$. We see further that if $e \in S, K(e) \in L\left(E, F^{\prime \prime}\right)$, since $q^{\prime \prime}(K(e) \cdot x)$ $\leqq|T|_{p-q} p(x)$.

Definition 1. We say $K$ from $S$ into $L\left(E, F^{\prime \prime}\right)$ is a weak regular Borel measure if for each $x \in E$ and $y^{\prime} \in F^{\prime}, \lambda(e)=\left\langle K(x) \cdot x, y^{\prime}\right\rangle$ is a regular Borel measure.

$K$ as defined above is a weak regular Borel measure, since $\lambda(e)$ $=\left\langle K(e) \cdot x, y^{\prime}\right\rangle=\left\langle T^{\prime \prime} \chi_{e} \cdot x, y^{\prime}\right\rangle=\left\langle\chi_{e} \cdot x, T^{\prime}\left(y^{\prime}\right)\right\rangle=\mu(e)_{\left(x, T^{\prime}\left(y^{\prime}\right)\right)}$, which is a regular Borel measure by construction. It is also obvious that $K$ is finitely additive. A further remark is that $K$ defined above agrees with the definition of $K$ given in [6] on the ring generated by compact $G_{\delta}^{\prime}$ 's. In [6] $K$ is such that $\left\langle K(e) \cdot x, y^{\prime}\right\rangle=\lim _{n}\left\langle T\left(f_{n} \cdot x\right), y^{\prime}\right\rangle$, where $\left\{f_{n}\right\}$ is a certain sort of bounded sequence of continuous functions converging pointwise to $\chi_{e}$. But

$\lim _{n}\left\langle T\left(f_{n} \cdot x\right), y^{\prime}\right\rangle=\lim _{n}\left\langle f_{n} \cdot x, T\left(y^{\prime}\right)\right\rangle=\lim _{n} \int f_{n} d \mu_{\left(x, T^{\prime}\left(y^{\prime}\right)\right)}=\mu(e)_{\left(x, T^{\prime}\left(y^{\prime}\right)\right),}$

by the Lebesgue dominated convergence theorem. But $\mu(e)_{\left(x, T^{\prime}\left(y^{\prime}\right)\right)}$ $=\left\langle\chi_{e} \cdot x, T^{\prime}\left(y^{\prime}\right)\right\rangle=\left\langle T^{\prime \prime}\left(\chi_{e} \cdot x\right), y^{\prime}\right\rangle$.

Definition 2. If $K$ is a finitely additive set function from $S$ into $L\left(E, F^{\prime \prime}\right)$, we say $f \in C(H, E)$ is integrable with respect to $K$ provided there exists a $y^{\prime \prime} \in F^{\prime \prime}$ such that given any $q^{\prime \prime}$ on $F^{\prime \prime}$, and $\epsilon>0$, there exists a partition $P_{0}$ of $H$ such that if $P=\left\{e_{1}, \cdots, e_{n}\right\}$ is any refinement of $P_{0}$, and $e_{i} \in S$ for all $i$, then $q^{\prime \prime}\left(y^{\prime \prime}-\sum_{i=1}^{n} K\left(e_{i}\right) x_{i}\right)<\epsilon$ for any choice of $x_{i}$ such that $x_{i} \in f\left(e_{i}\right)$ for all $i$. We say $y^{\prime \prime}=\int d K f$.

Definition 3. We say $P=\left\{e_{1}, \cdots, e_{n}\right\}$ is an $f-\epsilon-p$ partition of $H$ provided that $p\left(x_{1}-x_{2}\right)<\epsilon$ whenever $x_{1}, x_{2} \in f\left(e_{i}\right)$ for some $i$.

We now strengthen Lemma 1.

Lemma 2. If $f \in C(H, E)$,

$$
p^{\prime \prime}\left(f-\sum_{i=1}^{n} \chi_{e_{i}} \cdot x_{i}\right) \leqq \max _{t \in H} \quad p\left(f(t)-\sum_{i=1}^{n} \chi_{e_{i}}(t) x_{i}\right) .
$$


Proof. We first prove the representation theorem for the case when $F$ is the scalars. If $T=f^{\prime} \in C^{\prime}(H, E)$, then $K$ is of bounded variation so $\int d K f$ exists $\forall f \in C(H, E)$ since the scalars are complete. Also, it is easy to see from the definition of $K$ that $\left\langle f \cdot x, f^{\prime}\right\rangle=\int d K(f \cdot x), \forall f$ $\in C(H), \forall x \in E$. The linear span of the functions $f \cdot x, f \in C(H), x \in E$ are dense in $C(H, E)$, so $\left\langle f, f^{\prime}\right\rangle=\int d K f \forall f \in C(H, E)$, and $|f|_{p-q}=V_{p-q^{\prime \prime}}$ where $q$ is the absolute value. So for

$$
\begin{aligned}
f^{\prime} & \in B_{p}^{0}, p^{\prime \prime}\left(f-\sum_{i=1}^{n} \chi_{e_{i}} \cdot x_{i}\right) \\
& =\sup _{f^{\prime} \in B_{\boldsymbol{p}}^{0}}\left|\int d K\left(f-\sum_{i=1}^{n} \chi_{e_{i}} \cdot x_{i}\right)\right| \leqq V_{p-q^{\prime \prime}} \sup _{t \in H} p\left(f(t)-\sum_{i=1}^{n} \chi_{e_{i}}(t) \cdot x_{i}\right) \\
& =\sup _{t \in H} p\left(f(t)-\sum_{i=1}^{n} \chi_{e_{i}}(t) \cdot x_{i}\right) .
\end{aligned}
$$

We should remind the reader that we are thinking of $f \in C^{\prime \prime}(H, E)$ by the canonical imbedding.

We now state and prove our representation theorem.

Theorem 1. Given any continuous linear transformation $T$ from $C(H, E)$ into $F$ there exists a unique finitely additive set function $K$ from the Borel sets $S$ into $L\left(E, F^{\prime \prime}\right)$ such that $K$ is a weak regular Borel measure of finite $p-q^{\prime \prime}$ variation, and $\int d K f$ exists and $T(f)$ $=\int d K f, \forall f \in C(H, E)$, and $|T|_{p-q}=V_{p-q^{\prime \prime}}$.

Conversely, if $K$ is a finitely additive set function from $S$ into $L\left(E, F^{\prime \prime}\right)$ such that $K$ is of finite $p-q^{\prime \prime}$ variation, and $\int d K f$ exists and is in $F \forall f \in C(H, E)$ then $T(f)=\int d K f$ defines a continuous linear transformation from $C(H, E)$ into $F$.

Proof. If $T$ is $p-q$ related and $P$ is an $f-\epsilon-p$ partition of $H$,

$$
\begin{aligned}
q^{\prime \prime}\left(T(f)-\sum_{i=1}^{n} K\left(e_{i}\right) \cdot x_{i}\right) & =q^{\prime \prime}\left(T^{\prime \prime}\left(f-\sum_{i=1}^{n} \chi_{e_{i}} \cdot x_{i}\right)\right) \\
& \leqq|T|_{p-q} p^{\prime \prime}\left(f-\sum_{i=1}^{n} \chi_{e_{i}} \cdot x_{i}\right) \leqq|T|_{p-q} \epsilon,
\end{aligned}
$$

by Lemma 2 .

So $\int d K f$ exists and equals $T(f) \forall f \in C(H, E)$. Actually $\int d K f$ equals the canonical image of $T(f)$ in $F^{\prime \prime}$.

If $W$ is any other weak Borel measure of bounded $p-q^{\prime \prime}$ such that $T(f)=\int d W f$, then $\left\langle T(f \cdot x), y^{\prime}\right\rangle=\int f d \lambda$ where $\lambda(e)=\left\langle W(e) \cdot x, y^{\prime}\right\rangle$. But 


$$
\left\langle T(f \cdot x), y^{\prime}\right\rangle=\left\langle f \cdot x, T^{\prime}\left(y^{\prime}\right)\right\rangle=\int f d \mu_{\left(x, T^{\prime}\left(y^{\prime}\right)\right)}
$$

so

$$
\begin{aligned}
\left\langle W(e) x, y^{\prime}\right\rangle & =\lambda(e)=\mu_{\left(x, T^{\prime}\left(y^{\prime}\right)\right)}(e)=\left\langle\chi_{e} \cdot x, T^{\prime}\left(y^{\prime}\right)\right\rangle \\
& =\left\langle T^{\prime \prime}\left(\chi_{0}\right) \cdot x, y^{\prime}\right\rangle=\left\langle K(e) \cdot x, y^{\prime}\right\rangle .
\end{aligned}
$$

Thus $K$ is unique.

The converse is trivial.

REMARK 1. If one considers the case when $F$ is semireflexive, then one has the theorem that $T$ is a continuous linear transformation of $C(H, E)$ into $F$ if and only if there exists a unique weak regular Borel measure $K$ from $S$ into $L(E, F)$ of bounded $p-q$ variation such that $T(f)=\int d K f$, where the convergence of the integral is in the topology $\epsilon^{\circ \circ}$ of $F^{\prime \prime}$, over Borel partitions. (The proof is similar to [6, Proposition 4.3 , p. 255].)

REMARK 2. In the special case when $E$ and $F$ are linear normed spaces one notices that the $\epsilon^{\circ 0}$ topology is the norm topology of $F^{\prime \prime}$. Thus one has the theorem that if $T$ is a continuous linear transformation from $C(H, E)$ into $F$ then there exists a unique $K$ from $S$ into $L\left(E, F^{\prime \prime}\right)$ such that $K$ is a weak Borel measure, $K$ is of bounded variation, $T(f)=\int d K f$, where the convergence of the integral is in the norm convergence of $F^{\prime \prime}$ over Borel partitions. Also $\|T\|=V(K)$, variation of $K$.

3. A special case. If $E=$ scalars and $F=L(X, Y)$ Theorem 1 gives $K(e) \in L^{\prime \prime}(X, Y)$, but one would like to have $K(e) \in L\left(X, Y^{\prime \prime}\right)$. This is done in Theorem 2.

THEOREM 2. Given any continuous linear transformation $T$ from $C(H)$ into $L(X, Y)$ then there exists a unique weak regular Borel measure $K$ from $S$ into $L\left(X, Y^{\prime \prime}\right)$ where $K$ is of bounded variation $T(f)=\int d K f, \forall f$ $\in C(H)$, and $\|T\|=V(K)$.

Proof. Theorem 1 gives $T^{\prime \prime}\left(\chi_{e}\right) \in L^{\prime \prime}(X, Y)$ so we construct a map $\theta$ from $L^{\prime \prime}(X, Y)$ into $L\left(X, Y^{\prime \prime}\right)$. If $x \in X, y^{\prime} \in Y^{\prime}$ define $\left(x, y^{\prime}\right)$ $\in L^{\prime}(X, Y)$ by $\left(x, y^{\prime}\right)(v)=\left\langle v(x), y^{\prime}\right\rangle \forall v \in L(X, Y)$. Then define $\theta\left(v^{\prime \prime}\right)$ for $v^{\prime \prime} \in L^{\prime \prime}(X, Y)$ by $\left\langle\theta\left(v^{\prime \prime}\right)(x), y^{\prime}\right\rangle=v^{\prime \prime}\left(\left(x, y^{\prime}\right)\right)$. It is easy to see $\theta$ is a norm decreasing linear map from $L^{\prime \prime}(X, Y)$ into $L\left(X, Y^{\prime \prime}\right)$.

Define $K(e)=\theta\left(T^{\prime \prime}\left(\chi_{e}\right)\right)$. Then 


$$
\begin{aligned}
\| T(f) & -\sum_{i=1}^{n} f\left(t_{i}\right) K\left(e_{i}\right) \| \\
& =\left\|T(f)-\theta T^{\prime \prime}\left(\sum_{i=1}^{n} \chi_{e_{i}} f\left(t_{i}\right)\right)\right\|=\left\|\theta T^{\prime \prime}\left(f-\sum_{i=1}^{n} \chi_{e_{i}} \cdot x_{i}\right)\right\| \\
& \leqq\|T\| \sup _{t \in H}\left|f(t)-\sum_{i=1}^{n} \chi_{e_{i}} \cdot f\left(t_{i}\right)\right| .
\end{aligned}
$$

So we see $T(f)=\int d K f$. (Actually $\int d K f$ equals the element of $L\left(X, Y^{\prime \prime}\right)$ obtained by composing the map $T(f)$ with the canonical imbedding of $Y$ into $Y^{\prime \prime}$.)

REMARK 3. In the case $Y$ is reflexive we have $T$ is a continuous linear map of $C(H)$ into $L(X, Y)$ if and only if there exists a unique weak regular Borel measure $K$ with values in $L(X, Y)$ of bounded variation such that $T(f)=\int d K f, \forall f \in C(H)$.

4. Vector valued translation invariant $T$. Let us assume $H$ is a compact topological group and let $E$ and $F$ be linear normed spaces with $F$ complete. Suppose $T$ is a continuous linear transformation of $C(H, E)$ into $F$ with the property that $T\left(f_{t}\right)=T(f)$, where $f_{t}(z)$ $=f(t+z)$. We say $T$ is left-translation-invariant. Let $M$ be the space of all left-translation-invariant continuous linear transformations from $C(H, E)$ into $F$. We characterize $M$.

TheOREM 3. $M$ is isometrically isomorphic to $L(E, F)$, where $T \in M$ corresponds to $v \in L(E, F)$ if and only if $T(f)=\int v(f) d \mu$ for all $f \in C(H, E)$, where $v(f) \in C(H, F)$ is defined by $v(f)(t)=v(f(t))$, and $\mu$ is left-translation-invariant Haar measure normalized by $\mu(H)=1$.

Proof. For simplicity we shall assume the field is the real field; similar arguments prove the result for the complex case.

Let us assume $T \in M$. We shall show $K(e) \cdot x=v(x) \mu(e)$, where $v(x)=T\left(f_{0} \cdot x\right)$, where $f_{0}$ is the function that is identically one on $H$.

Consider $\phi(f)=\left\langle T f \cdot x, y^{\prime}\right\rangle, \phi \in C(H)$, and $\phi$ is left-translationinvariant. So by $[7,15 \mathrm{~A}$, p. 40$]$ we see there exist positive linear functionals $\phi_{1}$ and $\phi_{2}$ such that $\phi=\phi_{1}-\phi_{2}$. By considering the construction one sees $\phi_{1}$ and $\phi_{2}$ are translation invariant. By $[7,290$, p. 115] there exist constants $\lambda_{1}$ and $\lambda_{2}$ such that $\phi_{1}(f)=\lambda_{1} \int f d \mu, \phi_{2}(f)$ $=\lambda_{2} \int f d \mu, \forall f \in C(H)$, where $\mu$ is left-invariant-Haar-measure normalized by $\mu(H)=1$. Hence $\phi(f)=\lambda\left(y^{\prime}, x\right) \int f d \mu$ for some constant $\lambda\left(y^{\prime}, x\right)$. Thus $\left\langle T f \cdot x, y^{\prime}\right\rangle=\lambda\left(y^{\prime}, x\right) \int f d \mu$. Let $f=f_{0}$ and we see $\left\langle v(x), y^{\prime}\right\rangle$ $=\lambda\left(y^{\prime}, x\right)$. Thus $T(f \cdot x)=v(x) \int f d \mu, \forall f \in C(H)$ and $\forall x \in F$. So $\left\langle T(f \cdot x), y^{\prime}\right\rangle=\left\langle v(x), y^{\prime}\right\rangle\left(\int f d \mu\right)$, so by definition $\left\langle K(e) \cdot x, y^{\prime}\right\rangle=\left\langle v(x), y^{\prime}\right\rangle$ $\cdot \mu(e)$, or $K(e) \cdot x=\mu(e) v(x)$. 
By Theorem $1 T(f)=\int d K f=\int v(f) d \mu$. (Notice we didn't use completeness of $F$.) One can easily show $\|T\|=\|v\|$.

It is obvious that if $v \in L(E, F)$ then $T$ defined by $T(f)=\int v(f) d \mu$ is in $M$, and $T(f \cdot x)=v(x) \int f d \mu$, so $v(x)=T\left(f_{0} \cdot x\right)$. This shows uniqueness and completes the proof.

REMARK 4. In [3, p. 416] it is remarked that it is an unsolved problem to determine when $K(e) \in L(E, F)$ as opposed to $K(e) \in L\left(E, F^{\prime \prime}\right)$. We see from Theorem 3 that $T$ translation invariant is a very strong but interesting sufficient condition.

\section{BIBLIOGRAPHY}

1. J. Batt and H. König, Darstellung linearer transformationen durch vektorwertige Riemann-Stieltjes-Integrale, Arch. Math. 10 (1959), 273-287. MR 21 \#7423.

2. R. Bartle, N. Dunford and J. Schwartz, Weak compactness and vector measures, Canad. J. Math. 7 (1955), 289-305. MR 16, 1123.

3. N. Dinculeanu, Vector measures, Internat. Series of Monographs in Pure and Appl. Math., vol. 95, Pergamon Press, New York, 1967. MR 34 \#6011b.

4. N. Dunford and J. Schwartz, Linear operators. I: General theory, Pure and Appl. Math., vol. 7, Interscience, New York, 1958. MR 22 \#8302.

5. C. Foias and I. Singer, Some remarks on the representation of linear operators in spaces of vector-valued continuous functions, Rev. Math. Pures Appl. 5 (1960), 729-752. MR 24 \#A1618.

6. R. K. Goodrich, A Riesz representation theorem in the setting of locally convex spaces, Trans. Amer. Math. Soc. 131 (1968), 246-258. MR 36 \#5731.

7. L. H. Loomis, An introduction to abstract harmonic analysis, Van Nostrand, Princeton, N. J., 1953. MR 14, 883.

8. I. Singer, Sur les applications lineaires integrales des espaces de fonctions continues. I, Rev. Math. Pures Appl. 4 (1959), 391-401. MR 22 \#5883.

9. - Sur les applications linéaires intégrales des espaces de fonctions continues d valeurs vectorielles, Acta. Math. Acad. Sci. Hungar. 11 (1960), 3-13. MR 22 \#5884.

10. F. Riesz, Sur les operations fonctionelles linéaires, C. R. Acad. Sci. Paris 149 (1909), 974-977.

11. K. Swong, A representation theory of continuous linear maps, Math. Ann. 155 (1964), 270-291; errata, ibid. 157 (1964), 178. MR 29 \#2642.

12. D. Tucker, A representation theorem for a continuous linear transformation on a space of continuous functions, Proc. Amer. Math. Soc. 16 (1965), 946-953. MR 33 \#7865.

13. D. Uherka, A Riesz representation theorem, Ph.D. Thesis, University of Utah, Salt Lake City, 1964.

14. S. Wayment and D. Tucker, Absolute continuity and the Radon theorem (to appear).

UNIVERSITY OF Colorado 